Research Article

\title{
Examining the Effector Mechanisms of the Feishu Acupoint (BL13) in the Treatment of Pneumonia Based on Systematic Acupuncture and Moxibustion Research
}

\author{
Yan Xu, ${ }^{1}$ Jiali Cai, ${ }^{1}$ Weibin Li, ${ }^{2}$ Jingkun Miao, ${ }^{3}$ Yan Mei, ${ }^{2}$ Xinna Wang, ${ }^{1}$ Hanying Xu, \\ Qixiong Chen $\mathbb{D}^{2},{ }^{2}$ Fang Liu $\mathbb{D}^{2}, 2$ and Hongtao Cui $\mathbb{D}^{2,4}$ \\ ${ }^{1}$ Changchun University of Chinese Medicine, Changchun 130117, China \\ ${ }^{2}$ Chongqing Hospital of Traditional Chinese Medicine, Chongqing 400021, China \\ ${ }^{3}$ Newborn Screening Center, Chongqing Health Center for Women and Children, Chongqing 401332, China \\ ${ }^{4}$ Chongqing Medical University, Chongqing 400016, China
}

Correspondence should be addressed to Qixiong Chen; 13193039866@126.com,Fang Liu; 892677702@qq.com, and Hongtao Cui; 1070306132@qq.com

Received 27 January 2021; Revised 12 June 2021; Accepted 19 June 2021; Published 5 July 2021

Academic Editor: Hongcai Shang

Copyright @ 2021 Yan Xu et al. This is an open access article distributed under the Creative Commons Attribution License, which permits unrestricted use, distribution, and reproduction in any medium, provided the original work is properly cited.

\begin{abstract}
Background. Pneumonia is a serious global health problem. In traditional Chinese medicine, acupuncture or moxibustion is used to directly stimulate select acupoints on the surface of the human body and produce physical stimulation to further stimulate regulatory functions in the body, strengthening bodily resistance, eliminating disease, and adjusting the viscera. However, this Chinese medicine knowledge does not include the specific mechanisms of action or targets of acupoints. Therefore, an in-depth research is needed. Methods. An acupoint-element database was constructed, and the target elements of the Feishu point were screened. The UniProtSwiss-Prot sublibrary was used to obtain correct gene name information. The National Center for Biotechnology Information (NCBI) Gene Expression Omnibus (GEO) database and GEO2R were used to analyze differentially expressed genes in pneumonia. The STRING database was used to analyze interactions, construct a network of the Feishu point efficacy system in pneumonia, and elucidate the mechanisms of action. Results. The Feishu point comprises 34 elements in total. The protein interaction analysis has 38 nodes and 115 edges. The Feishu point efficacy system-pneumonia system network shows that cytokine signaling in the immune system, signaling by interleukins (ILs), IL-4 and IL-13 signaling, and the immune system may be related to immunity and inflammation. The Feishu point efficacy system regulating pneumonia showed that FCER2, IL4R, FASLG, TGFB1, IL6R, STAT6, IL1B, CASP3, IL5RA, IL2RB, MYD88, SQSTM1, IL12RB1, IFNGR1, ADAM17, and CDH1 are the main targets. Conclusion. From the perspective of systematic acupuncture and moxibustion, the Feishu point regulates cytokine signaling in the immune system, signaling by ILs, IL-4 and IL-13 signaling, and the immune system by targeting FCER2, IL4R, FASLG, TGFB1, IL6R, STAT6, IL1B, CASP3, IL5RA, IL2RB, MYD88, SQSTM1, IL12RB1, IFNGR1, ADAM17, and CDH1, thereby regulating pneumonia.
\end{abstract}

\section{Background}

The Feishu point (BL13) was first described in Lingshu Backshu. It belongs to the bladder meridian (BL) and is the Back-Shu point of the lung. This point is located 1.5 inches beside and below the spinous process of the third thoracic vertebra on the back. The indications for Feishu point stimulation include pulmonary symptoms such as cough, expectoration, wheezing, and shortness of breath. Acupoint massage [1], applicator therapy [2,3], and iontophoresis [4] at the Feishu point can shorten the time to cough improvement and reduce cough symptom scores, inflammatory indicator levels, and adverse reaction rates in patients with pneumonia. Moxibustion at the Feishu point can regulate fatigue and muscle aches in patients with severe acute respiratory syndrome coronavirus 2 (SARS-CoV-2) [5]. The efficacy of the Feishu point in the treatment of lung diseases has been verified for thousands of clinical applications, but studies on its specific mechanisms of action are rare. Because of the modernization and globalization of 
traditional Chinese medicine, the mechanisms of action of the Feishu point must be addressed.

Investigations of the mechanisms of action of acupoints must first explain the nature of meridians and collaterals. However, controversy regarding the nature of meridians and collaterals remains. Some scholars working from the perspective of modern medicine consider meridians and collaterals to be complicated three-dimensional structures composed of nerves, blood vessels, muscles, tendons, and fasciae. Acupuncture and moxibustion stimulate acupoints to cause changes in liquid components, nerve conduction, and physical properties in local tissues, which result in the alleviation or cure of the disease condition in the human body [6, 7]. However, some scholars disagree and consider that the understanding of the nature of "meridians and collaterals" is inseparable from the specific lifestyle, cognitive orientation, and language framework of traditional Chinese medicine, namely, traditional Chinese medicine culture and theory. The evolution of modern medical explanations and research methods deviates from the original meaning of the original classics [8]. The present study considered that current explorations of the philosophical theory of traditional Chinese medicine and the development of science and technology cannot resolve the issue of the nature of meridians and collaterals. However, methods that indirectly elucidate the mechanisms of action of acupoints may be applied.

Proteins are important constituents of all cells and tissues in the human body. All substances with physiological activities in the human body, such as amines, neurotransmitters, polypeptide hormones, antibodies, enzymes, nuclear proteins, and proteins that play "carrier" roles in cell membranes and in blood, are inseparable from proteins. Proteins play very important roles in the regulation of physiological functions and the maintenance of metabolism. Acupoint stimulation produces positive and negative regulatory effects in the body. The external presentation is disease improvement and recovery, and the internal presentation is an increase or decrease in protein levels. Therefore, the direct detection of changes in protein levels in the body bypasses the "black box" of the mechanisms of action of acupoints and indirectly elucidates these mechanisms (Figure 1).

Pneumonia is a serious global health problem [9], and it is the most common cause of death from infectious diseases worldwide, causing approximately 3.5 million deaths annually [10]. South Asia and Sub-Saharan Africa have the most severe pneumonia infections because of the economy and lack of medical care. Additionally, most deaths from pneumonia occur in children because of their unique physiological and pathological factors [11]. Acupuncture and moxibustion directly stimulate select acupoints on the surface of the human body and produce physical stimulation to further stimulate regulatory functions in the body, including the dredging of meridians and collaterals, the coordination of qi and blood, the strengthening of bodily resistance, the elimination of disease, and adjustment of the viscera. Because no special apparatus or equipment is required, acupuncture and moxibustion may be used as major methods for treating pneumonia in areas with scarce resources.

In summary, this study used systematic acupuncture and moxibustion to investigate the specific mechanisms of action of the Feishu point for regulating pneumonia. The results provide new ideas and methods for the globalization and moderation of acupuncture and moxibustion.

\section{Methods}

2.1. Construction of the Acupoint-Element Database. We performed a massive search of the literature in the China National Knowledge Infrastructure (CNKI), the Wanfang database, the Chongqing VIP Information network, PubMed, and Web of Science and constructed an acupointelement (target) database for the 12 main meridians, conception vessels (CVs), and governor vessels (GVs) that included a total of 361 acupoints. Dedicated personnel rechecked, debugged, double-checked, and supplemented the results.

2.2. Retrieval of Target Elements of the Feishu Point. The Feishu point was retrieved from the acupoint-element (target) database, and a total of 34 target elements were obtained. Related information can be found on the website https://www.tcmmodel.com/feishu/. These target elements were entered into the UniProt database (https://www. uniprot.org/), one at a time for correction and conversion into gene names. UniProt is a nonredundant protein sequence database with the most complete sequence data and the most abundant annotated information worldwide. The more than 500,000 sequences in its Swiss-Prot sublibrary were manually reviewed and annotated. The correction databases used in this study used the Swiss-Prot sublibrary.

2.3. The Protein-Protein Interaction (PPI) Network and the Feishu Point Efficacy System Mechanism Network. The STRING database (https://string-db.org/) was used to search for interactions between proteins (gene names may be entered). It contains direct physical interactions between proteins and indirect functional correlations between proteins. The present study used the PPI network in STRING to evaluate elemental relationships. The median interaction score was 0.4 .

The interaction score was the standard for determining the connections of the PPI network. The score was obtained via examination of the predictive performance using the public reference set (KEGG database), with the real relationship as the standard. The calculation formula was as follows:

$$
Q=\log \left\{\frac{\left(N_{\text {together }} \cdot N_{\text {total }}\right)}{\left[\left(N_{\text {alone }_{1}}+1\right) \cdot\left(N_{\text {alone }_{2}}+1\right)\right]}\right\} .
$$

The diagram in this study concealed nodes that did not have interaction to ensure the reliability of the interactions in the PPI network. 


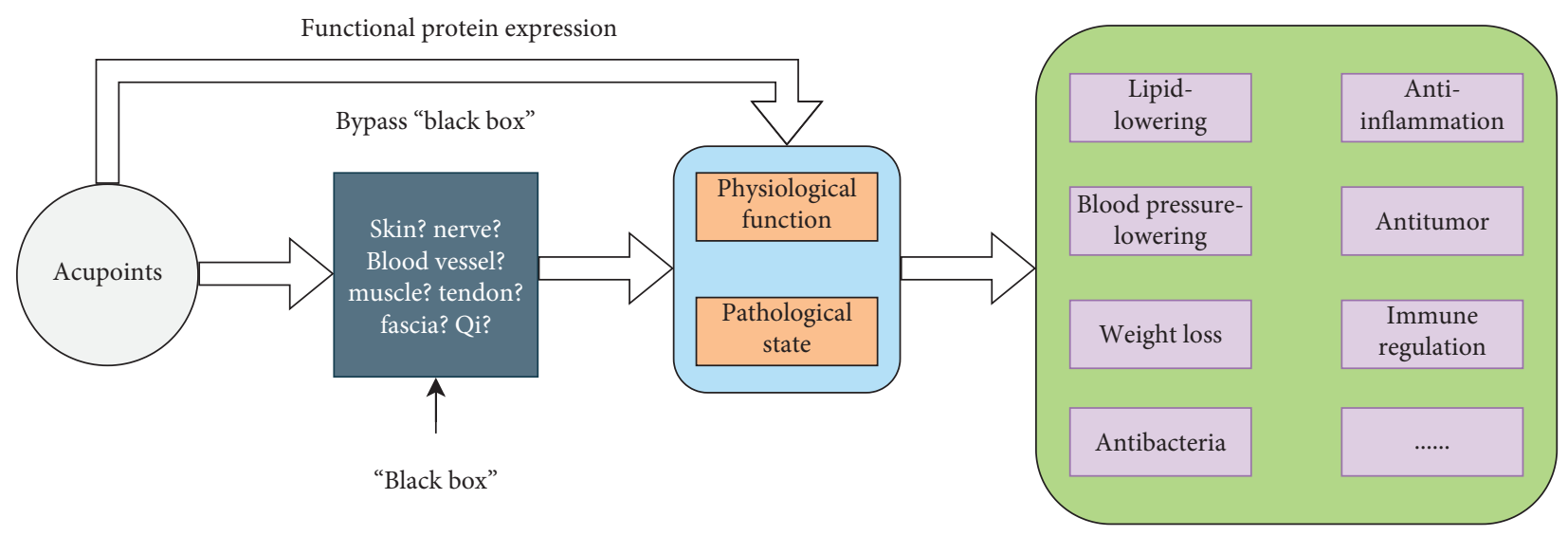

FIGURE 1: Road map of the mechanisms of action of acupoints.

2.4. Plotting the Network Diagram. The network of the interaction between the pneumonia efficacy system and pneumonia was plotted using Cytoscape (version 3.7.2). The degree value reflected the number of connections that a node in the network had with other nodes, which was the most intuitive parameter for determining the influence of a node. When the degree was larger, the influence was greater. Betweenness was the ratio of the closeness of nodes that passed through a certain node to the total closeness. When the betweenness was larger, the node was more important in the network. Closeness was the reciprocal of the average distance of the shortest paths between a node and other nodes in the network. Closeness considered the average length of the shortest paths between each node and other nodes. When a node was closer to other nodes, its closeness was higher.

\subsection{Differentially Expressed Genes in Pneumonia.} Differentially expressed genes were obtained from the National Center for Biotechnology Information (NCBI) Gene Expression Omnibus (GEO) database (https://www. ncbi.nlm.nih.gov/). The GEO is an online database that may be used to retrieve the gene expression data for any species. The GSE103119 data used in the present study contained the transcriptome data of 152 children with community-acquired pneumonia and 20 healthy children. The GEO2R online tool was used for analysis [12], and | $\operatorname{LogFC} \mid>1$ and $P<0.05$ were used as the standards for screening differentially expressed genes. Children were chosen as subjects because children are the main victims of pneumonia.

2.6. Reactome Pathways. Reactome (https://reactome.org/) is a biological database of various reactions and biological pathways in the human body that has annotated more than $70 \%$ of human proteins. This study used the Reactome database for the functional analysis. The false discovery rate (FDR) was defined as the percentage of all discoveries that were false. When the FDR was smaller, the result was more reliable. The formula was as follows:

$$
F D R=\frac{F P}{(F P+T P)},
$$

where FP (false positive) indicated that the detection result of a negative experimental sample was positive and TP (true positive) indicated that the detection result of a positive experimental sample was positive.

\section{Results}

3.1. Identification of Target Elements in the Feishu Point Efficacy System. The targets were the focus of the construction of the Feishu point efficacy system and were considered the basic routes by which acupoints exert their effects. The targets of the Feishu point were obtained via a database search using the search term "Feishu" (Table 1).

3.2. Correction of Target Element Names in the Feishu Point Efficacy System. The target element names in the database were obtained from the literature search, and the nomenclature of targets in the literature varied slightly according to the reagent company. The target elements were entered into the UniProt database one at a time to standardize the nomenclature. All genes were uniformly expressed as gene names (Table 2).

3.3. Construction of the Structure and Relationship of the Feishu Point Efficacy System. The STRING website was used to perform a protein interaction analysis of all targets of lung acupoints. Then, the network of relationships among the elements was built, and a structure-relation network was constructed (Figure 2 ). There were 38 nodes and 115 edges in the network. To extract the key elements in the network, calculations were performed based on degree, betweenness, and closeness. Elements with the higher-than-average degree, betweenness, and closeness values were considered key elements in the network (Table 3).

3.4. Construction of the Feishu Point Efficacy System Network. To describe the Feishu point efficacy system network in detail, Reactome pathway enrichment was used to define the 
Table 1: Target elements of the Feishu point.

\begin{tabular}{ll}
\hline Acupoint & Targets \\
\hline & LC3-II $[13,14], p 62[13]$, IL-6 [13, 15], Bax [16], Fas [16], FasL [16], Bcl-2 [16], IL-1 $\beta[17,18]$, E-cad [19], MyD88 [20], caspase \\
Feishu & 3 [20], p-gp [21, 22], IL-12 [23, 24], IL-4 [15, 23, 24], IgE [23], IL-2 [24, 25], IL-5 [24], IFN- $\gamma$ [15, 26, 27], TGF $\beta 1$ [25, 28], \\
point & $\beta$-catenin [29], MMP-7 [29], WISP-1 [29], Wnt3a [29], Beclin-1 [14], ACTH [15], CYP3A [22], TNF- $\alpha$ [22], sICAM-1 [25], \\
& CCL1 [30], CCR8 [30], STAT6 [30], ChAT [31], AChE [31], and mAChRs [31]
\end{tabular}

TABLE 2: Correction of target elements of the Feishu point.

\begin{tabular}{lc}
\hline Acupoint & Gene name \\
\hline $\begin{array}{l}\text { Feishu } \\
\text { point }\end{array}$ & MAP1LC3B, SQSTM1, IL6R, BAX, FAS, FASLG, BAD, IL1B, CDH1, MYD88, CASP3, ABCB5, IL12RB1, IL4R, FCER2, \\
& IL2RB, IL5RA, IFNGR1, TGFB1, CTNNBIP1, MMP7, CCN4, Wnt3a, BECN1, MC2R, Cyp3a2, ADAM17, sICAM-1, CCL1, \\
CCR8, STAT6, CHAT, AChE, and SLURP2
\end{tabular}

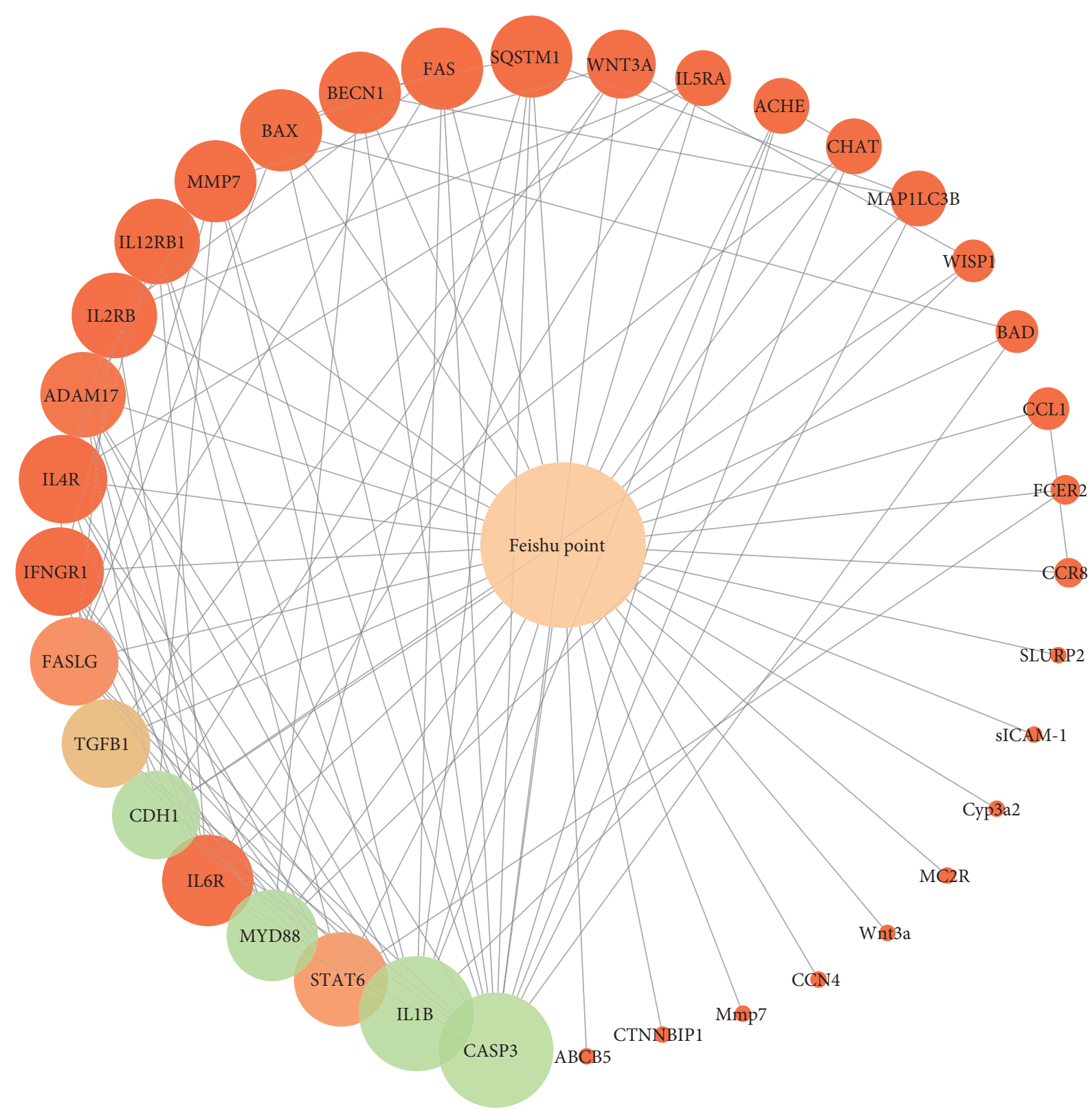

Figure 2: Relationship between the structure and efficacy of the Feishu point. The circle represents the degree, with larger circles corresponding to greater degrees. The color represents betweenness, with light green representing extensive betweenness and red representing limited betweenness. 
Table 3: Key elements of the Feishu point efficacy system.

\begin{tabular}{lccc}
\hline Gene name & Degree & Betweenness & Closeness \\
\hline CASP3 & 17 & 0.07995257 & 0.64912281 \\
IL1B & 17 & 0.06253873 & 0.64912281 \\
STAT6 & 10 & 0.01346287 & 0.578125 \\
MYD88 & 9 & 0.03216609 & 0.56923077 \\
IL6R & 9 & 0.00638972 & 0.55223881 \\
CDH1 & 8 & 0.03350434 & 0.56060606 \\
TGFB1 & 8 & 0.01788336 & 0.56060606 \\
FASLG & 8 & 0.0114984 & 0.55223881 \\
IFNGR1 & 8 & 0.00362446 & 0.56060606 \\
IL4R & 8 & 0.00296964 & 0.54411765 \\
ADAM17 & 7 & 0.00740502 & 0.55223881 \\
IL2RB & 7 & 0.00467134 & 0.53623188 \\
IL12RB1 & 7 & $9.26 E-04$ & 0.53623188 \\
MMP7 & 6 & 0.00372635 & 0.4625 \\
BAX & 6 & 0.0025025 & 0.53623188 \\
BECN1 & 6 & 0.00234163 & 0.54411765 \\
FAS & 6 & 0.00187688 & 0.53623188 \\
SQSTM1 & 6 & 0.00146575 & 0.54411765 \\
\hline
\end{tabular}

boundary of the system using the FDR parameter as the standard (Table 4).

\subsection{Differentially Expressed Genes in Pneumonia.} Differentially expressed genes in pneumonia were obtained from the GEO database. Differentially expressed genes were screened from healthy children and children with community-acquired pneumonia, and a total of 1074 genes were obtained (supplementary file (available here)).

3.6. Construction of the Feishu Point Efficacy SystemPneumonia System Network. Systems do not exist in isolation. Similar to elements, systems also have relationships and structures. Therefore, the Feishu point efficacy system may also have a relationship with the pneumonia system. This relationship would be the key point of interaction between the two systems, and all physiological and biological functions would be based on this relationship. The construction of the Feishu efficacy system-pneumonia system network was equivalent to artificially determining the boundary of the Feishu point efficacy system so that only the parts of the Feishu point efficacy system that were relevant to pneumonia could be studied. After establishing the correspondence between the Feishu point efficacy system and the differentially expressed targets in pneumonia, the Feishu point efficacy system-pneumonia network relationship diagram was constructed (Figure 3 ). The Feishu point efficacy system had 4 pathways that could function in pneumonia, including cytokine signaling in the immune system, signaling by interleukins (ILs), IL-4 and IL-13 signaling, and the immune system. These 4 pathways were all associated with immunity and inflammation.

3.7. Mechanism of Action of by which the Feishu Point Efficacy System Regulates Pneumonia. The roles of all of the proteins in the Feishu point efficacy system in the efficacy network were further examined. A median interaction score of 0.4 was used as the cutoff value for the plot shown in Figure 4. Sixteen proteins were involved in the mechanism by which the Feishu point efficacy system regulates pneumonia. FCER2, IL4R, FASLG, TGFB1, IL6R, STAT6, and IL1B were involved in the functions of all pathways, and CASP3, IL5RA, IL2RB, MYD88, SQSTM1, and IL12RB1 were involved in cytokine signaling in the immune system, IL signaling, and the immune system. IFNGR1 and ADAM17 were involved in cytokine signaling in the immune system and the immune system, and CDH1 was involved in the immune system.

\section{Discussion}

4.1. Theoretical Basis of Systematic Acupuncture and Moxibustion. The extensive application of network pharmacology, systems pharmacology, and integrated pharmacology fields in recent years has promoted the development of molecular biology and pharmacology-related subjects that integrate systems science theory. These methods and technologies have focused on interactions between the body and drugs at the overall level and have promoted the preliminary establishment of a theory of systematic traditional Chinese medicine [32, 33]. Against the backdrop of systematic traditional Chinese medicine theory, the present study proposed a concept of systematic acupuncture and moxibustion that could affect the mechanisms of action of acupoints, meridians, and collaterals. This concept considered systematic acupuncture and moxibustion as a multidisciplinary subject of epistemology, a methodology that uses systems science thinking and methods to understand acupoints, meridians, and collaterals. This emerging discipline uses systems engineering technology to elucidate microscopic and macroscopic structures and relationships and can be applied to the science and art of acupoints, meridians, and collaterals. It is also an interdisciplinary subject that integrates acupuncture and moxibustion with systems science to unravel the self-similarity, self-organization, and selfadaptability of systems and elucidate the inheritance, additivity, and emergence of overall functions. The basic concept of systematic acupuncture and moxibustion includes elements and the relationships among elements, structures, boundaries, and functions. In systems science, a system is a whole that interacts with the external environment and has specific capabilities [34]. Elements are basic factors that constitute a system. Relationships refer to relationships between elements at the same level. Structure refers to the relationships between elements at different levels. The boundary is the boundary between the inside and outside of a system. Functions refer to the characteristics, behavior, performance, and functions of interactions between the system and the external environment [35]. Therefore, the use of data mining technology and biological network technology to confirm elements, structures, boundaries, and functions from the perspective of systematic acupuncture and moxibustion may systematically explain the mechanisms of action of acupoints, meridians, and collaterals (Figure 5). 
TABle 4: Top 10 Reactome enrichment results for the Feishu point efficacy system.

\begin{tabular}{|c|c|c|c|}
\hline $\begin{array}{l}\text { Reactome } \\
\text { ID }\end{array}$ & Reactome description & FDR & Matched element \\
\hline $\begin{array}{l}\text { HSA- } \\
1280215\end{array}$ & $\begin{array}{c}\text { Cytokine signaling in the immune } \\
\text { system }\end{array}$ & $7.18 E-12$ & $\begin{array}{l}\text { ADAM17, CASP3, FASLG, FCER2, IFNGR1, IL12RB1, IL1B, IL2RB, } \\
\text { IL4R, IL5RA, IL6R, MYD88, SQSTM1, STAT6, and TGFB1 }\end{array}$ \\
\hline $\begin{array}{l}\text { HSA- } \\
449147\end{array}$ & Signaling by interleukins & $1.31 E-11$ & $\begin{array}{c}\text { CASP3, FASLG, FCER2, IL12RB1, IL1B, IL2RB, IL4R, IL5RA, IL6R, } \\
\text { MYD88, SQSTM1, STAT6, and TGFB1 }\end{array}$ \\
\hline $\begin{array}{l}\text { HSA- } \\
162582\end{array}$ & Signal transduction & $1.23 E-08$ & $\begin{array}{c}\text { ADAM17, BAD, BAX, CASP3, CCL1, CCR8, CDH1, CTNNBIP1, FAS, } \\
\text { FASLG, FCER2, IL2RB, IL5RA, IL6R, MC2R, MYD88, SQSTM1, } \\
\text { STAT6, TGFB1, and WNT3A }\end{array}$ \\
\hline $\begin{array}{l}\text { HSA- } \\
6785807\end{array}$ & $\begin{array}{l}\text { Interleukin-4 and Interleukin-13 } \\
\text { signaling }\end{array}$ & $3.34 E-08$ & FASLG, FCER2, IL1B, IL4R, IL6R, STAT6, and TGFB1 \\
\hline HSA-73887 & Death receptor signaling & $1.62 E-07$ & ADAM17, BAD, CASP3, FAS, FASLG, MYD88, and SQSTM1 \\
\hline $\begin{array}{l}\text { HSA- } \\
168256\end{array}$ & Immune system & $4.40 E$ & $\begin{array}{l}\text { ADAM17, CASP3, CDH1, FASLG, FCER2, IFNGR1, IL12RB1, IL1B, } \\
\text { IL2RB, IL4R, IL5RA, IL6R, MYD88, SQSTM1, STAT6, and TGFB1 }\end{array}$ \\
\hline $\begin{array}{l}\text { HSA- } \\
109581\end{array}$ & Apoptosis & $9.96 E-06$ & BAD, BAX, CASP3, CDH1, FAS, and FASLG \\
\hline $\begin{array}{l}\text { HSA- } \\
5357801\end{array}$ & Programmed cell death & $9.96 E-06$ & BAD, BAX, CASP3, CDH1, FAS, and FASLG \\
\hline $\begin{array}{l}\text { HSA- } \\
193704\end{array}$ & p75 NTR receptor-mediated signaling & $1.68 E-05$ & ADAM17, BAD, CASP3, MYD88, and SQSTM1 \\
\hline $\begin{array}{l}\text { HSA- } \\
5357769\end{array}$ & $\begin{array}{l}\text { Caspase activation via the extrinsic } \\
\text { apoptotic signaling pathway }\end{array}$ & 0.00032 & CASP3, FAS, and FASLG \\
\hline
\end{tabular}

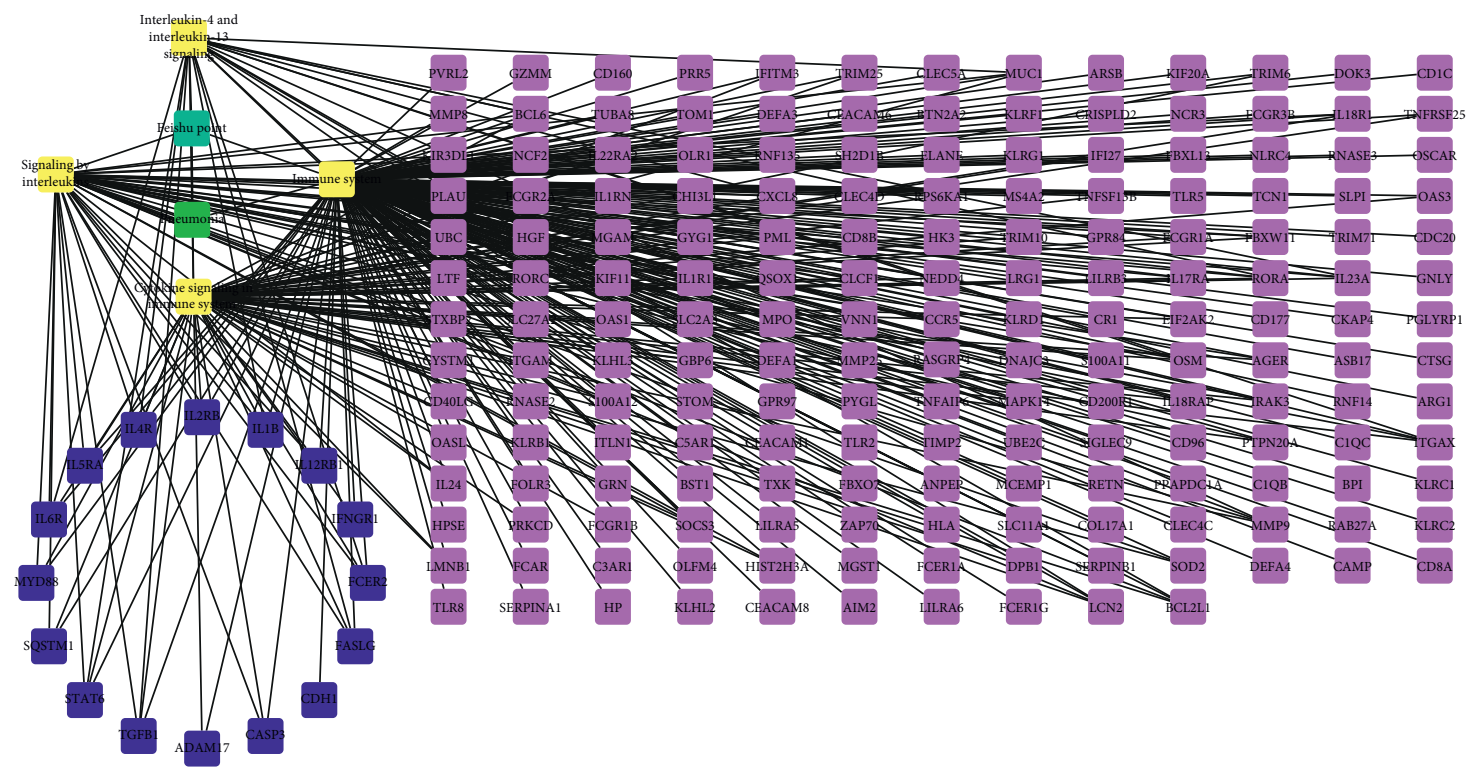

Figure 3: The Feishu point efficacy system-pneumonia system interaction network.

4.2. Investigation of the Mechanisms of Action of the Feishu Point for the Regulation of Pneumonia. The present study demonstrates that the Feishu point exerts therapeutic effects in pneumonia via cytokine signaling in the immune system, IL signaling, IL-4 and IL-13 signaling, and the immune system. Cytokines are peptide substances that are extensively present in the body. Cytokines are secreted by immune cells, such as macrophages, B lymphocytes, $\mathrm{T}$ lymphocytes, and mast cells, as well as endothelial cells, fibroblasts, and various stromal cells. Based on their biological functions, cytokines are divided into chemokines, interferons, ILs, lymphokines, and tumor necrosis factors (TNFs). Cytokines in body fluids or tissues regulate the development, differentiation, and function of immune cells at a lower level under normal conditions to maintain the balance of the cytokine network. Once the body encounters abnormalities, the balance between anti-inflammatory cytokines and proinflammatory cytokines is impaired, and large amounts of proinflammatory cytokines are produced. Cells and intracellular signaling pathways also change accordingly, resulting in inflammation and immune processes [36]. IL-4 is primarily produced by activated T helper 2 (Th2) cells and mast cells, and it plays a role in the production of Th2 cytokines and the immunoregulatory function of lymphocytes and macrophages. IL-4 blocks antibody-dependent cytotoxicity, inhibits the production 


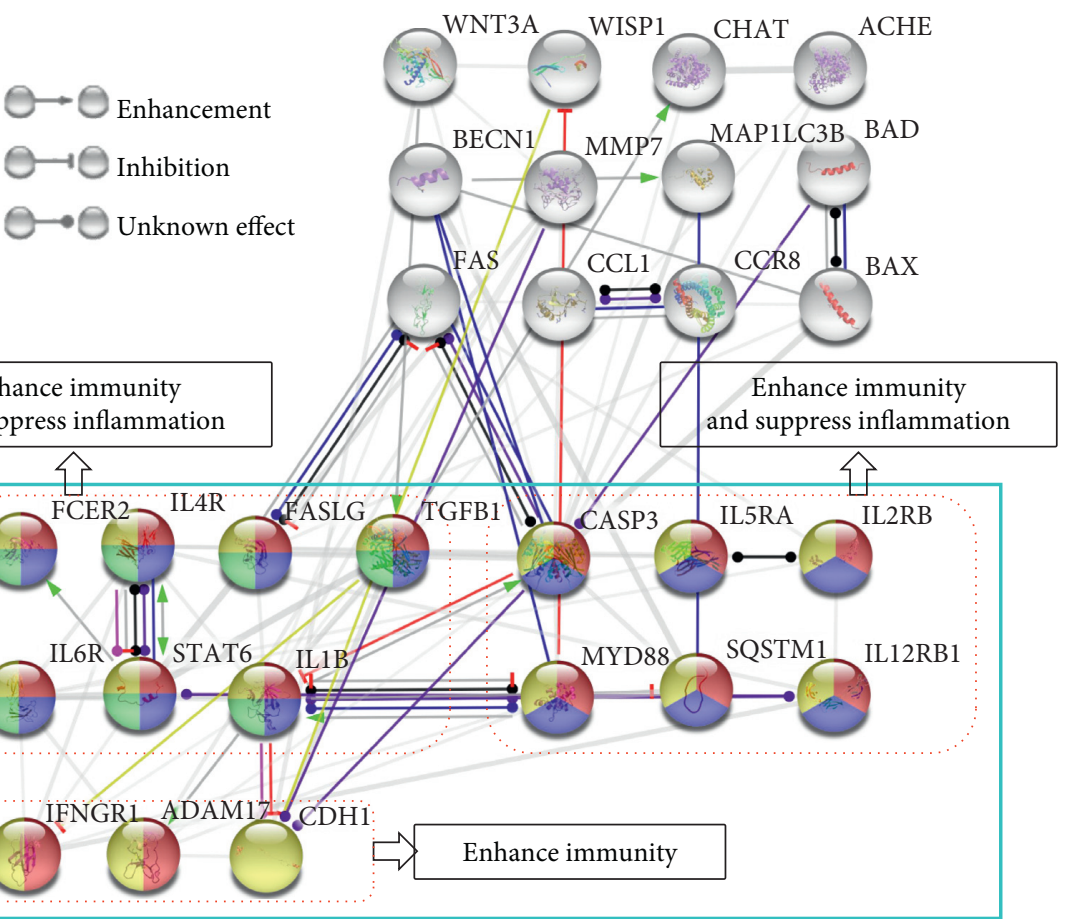

Figure 4: Diagram of the mechanisms of action of the Feishu point efficacy system in the treatment of pneumonia. Red indicates cytokine signaling in the immune system, blue indicates signaling by ILs, green indicates IL- 4 and IL-13 signaling, yellow indicates the immune system, and white indicates other systems.

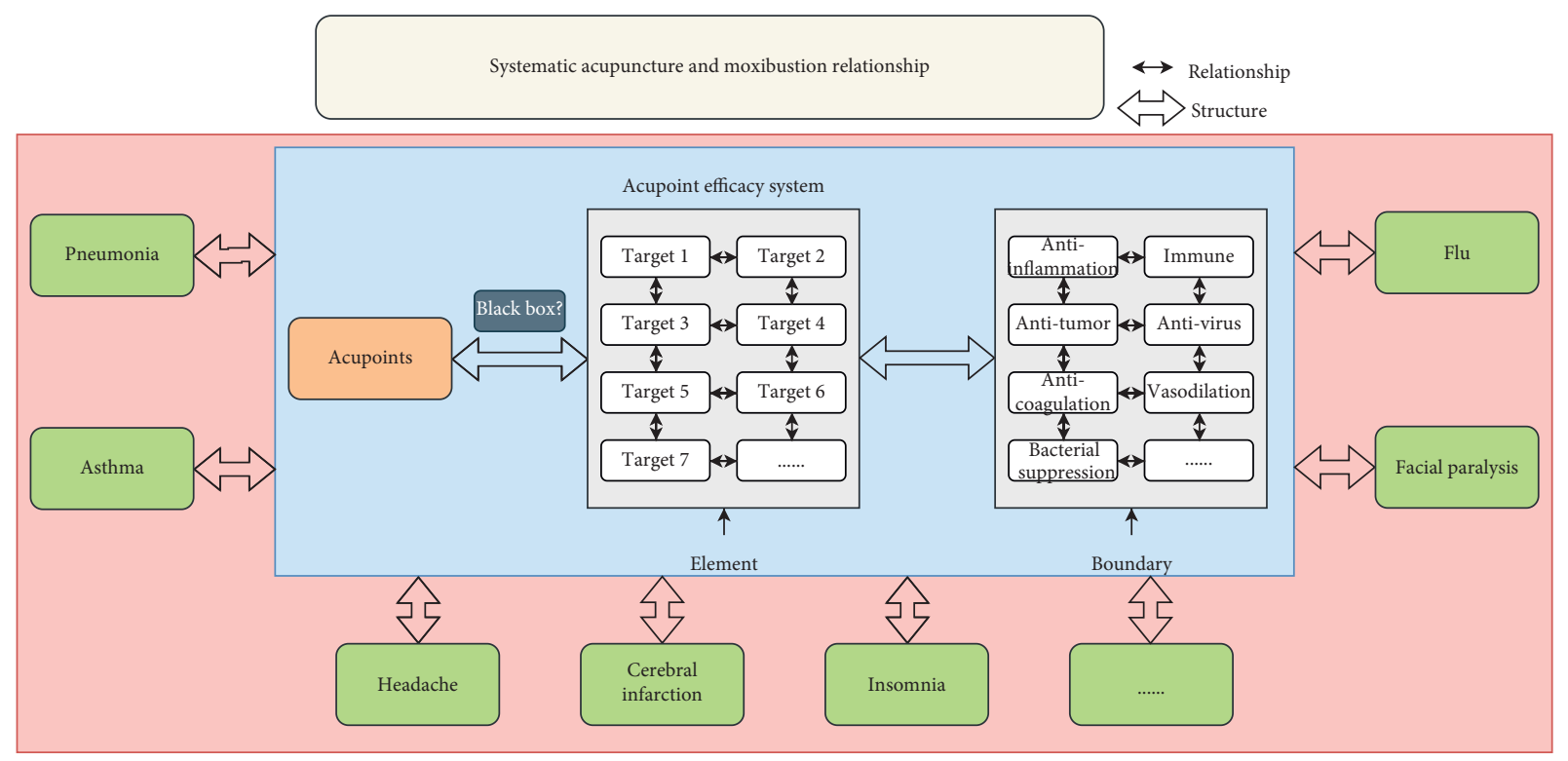

FIGURE 5: Systematic acupuncture and moxibustion research methods.

of IL- $1 \beta$, TNF- $\alpha$, prostaglandin E2, IL-6, IL-8, and nitric oxide, and induces B cells to produce IgG and IgE [37]. IL13 also downregulates the synthesis of TNF- $\alpha$, IL-1, and IL6 and promotes IgE synthesis [38]. Overall, as important cytokines, IL-4 and IL-13 play important roles in mitigating the development of inflammatory responses and increasing immunity. At the gene level, this study demonstrates that the Feishu point regulates cytokine signaling in the immune system, signaling by ILs, IL-4 and IL-13 signaling, and the immune system via FCER2, IL4R, FASLG, TGFB1, IL6R, STAT6, IL1B, CASP3, IL5RA, IL2RB, MYD88, SQSTM1, IL12RB1, IFNGR1, ADAM17, and CDH1 gene regulation to exert effects on the regulation of inflammation and immunity (Figure 6), thereby preliminarily elucidating the mechanisms of action of the Feishu point in treating pneumonia. 


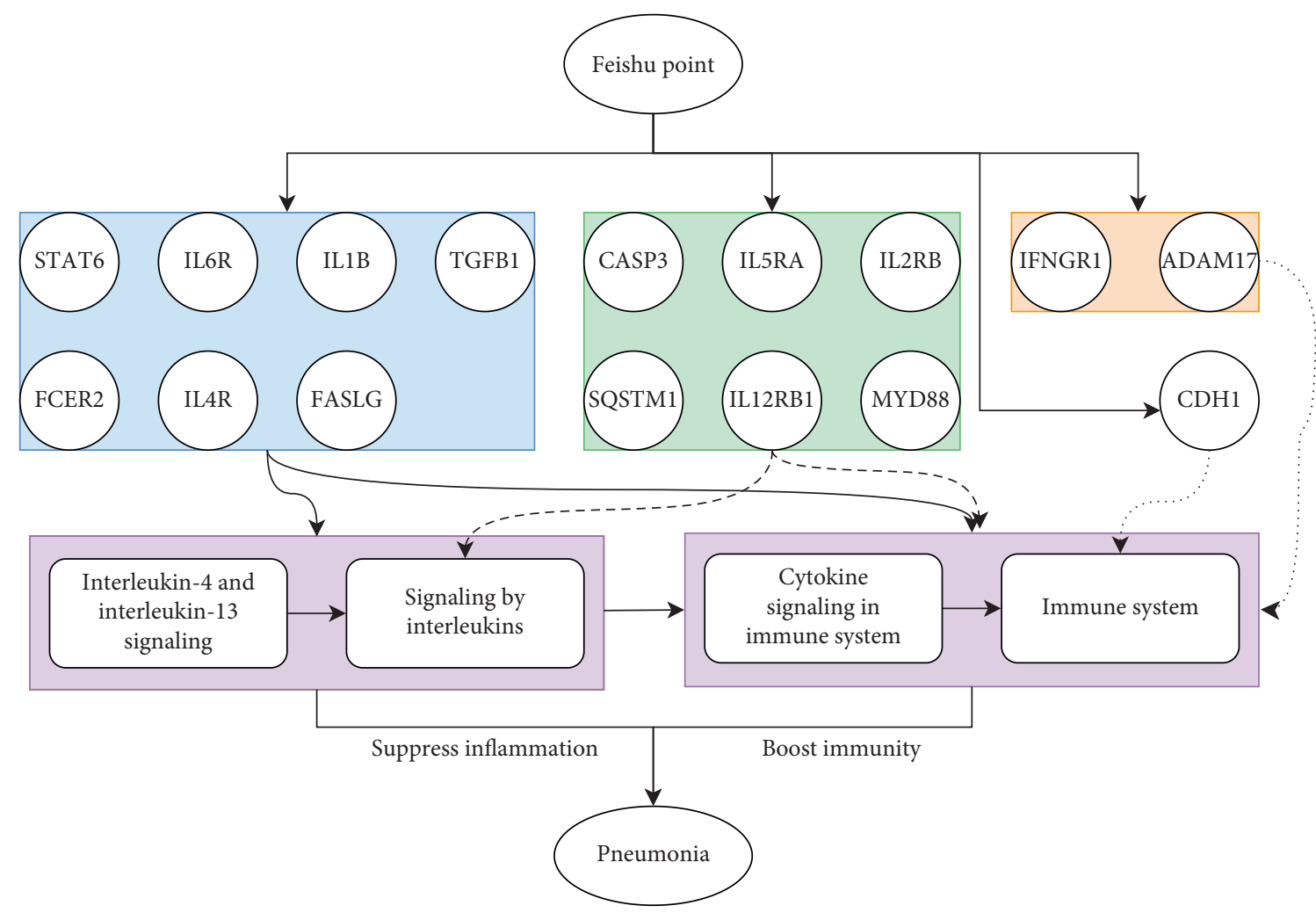

Figure 6: Mechanisms of action of the Feishu point in the regulation of pneumonia.

Our research has some clinical significance. It differs from previous clinical studies that focused on different methods, such as application [39], cupping [40], and acupoint injection [41] to stimulate the Feishu acupoint and achieve the treatment goal. Our research starts with the physiological and pathological mechanisms that occur after acupoints are stimulated and adds specific biological evidence supporting their clinical effectiveness. Furthermore, unlike the previously described single mechanism [42, 43], we present a more comprehensive description of the mechanism through which lung acupoints regulate pneumonia.

4.3. Feishu Point Treatment System. The Feishu point is a Back-Shu point on the bladder meridian (BL). It is located 1.5 inches beside and below the spinous process of the third thoracic vertebra and corresponds to the lung. The Feishu point is also the Back-Shu point of the lung. A complete review of the Feishu point treatment system based on existing studies showed very good efficacy of the Feishu point for the treatment of lung diseases, including cough
[44], pneumonia with dyspnea and cough [45, 46], asthma [47], acute lung injury [48], allergic rhinitis [49], chronic obstructive pulmonary disease [50], lung cancer [51, 52], acute tonsillitis [49], and bronchiolitis [53]. The Feishu point efficacy system is also used to treat heart diseases, such as chronic heart failure [54], and skin diseases, such as chronic urticaria [55], shingles [56], abdominal urticaria [57], and acne [58]. To specifically elucidate the Feishu point efficacy system, it was considered as a whole according to systematic acupuncture and moxibustion theory and the evidence presented above [44-58] and plotted as a diagram (Figure 7). The diagram shows that the Feishu point treatment system is a complicated network that includes various diseases. This point plays a role in lung diseases, heart diseases and some skin diseases. This treatment system completely satisfies the theoretical bases of "all meridians and vessels converge in the lung to assist the heart in promoting blood circulation" and "the lung connects with the skin," and it is a specific presentation of traditional Chinese medicine theory.

In summary, the elucidation of the therapeutic effect of the Feishu point from an overall perspective via the construction of the Feishu point treatment system met the basic 


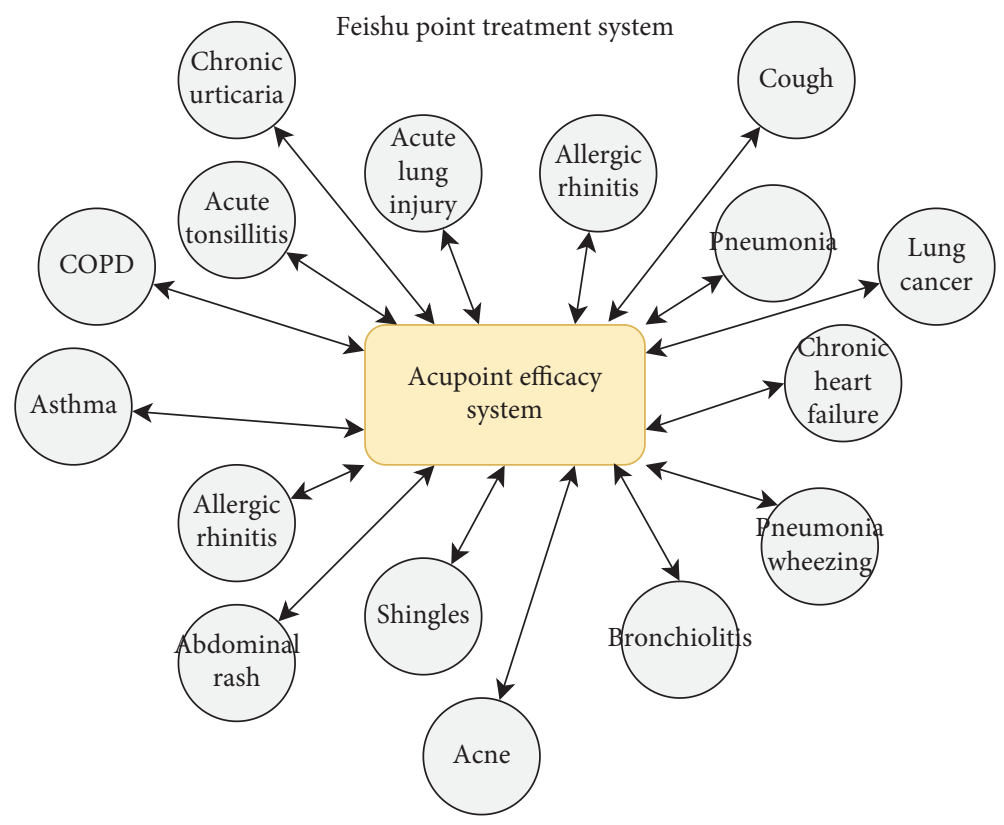

FIgURE 7: The Feishu point treatment system.

characteristics of traditional Chinese medicine theory and provided scientific evidence for studies on the elucidation of the systematic functions and modernization of acupoints.

\section{Conclusion}

(1) From the perspective of systematic acupuncture and moxibustion, this research explored the specific mechanism by which the Feishu point regulates pneumonia and provided new ideas for the development of acupuncture and moxibustion.

(2) The Feishu point regulates cytokine signaling in the immune system, signaling by ILs, IL-4, and IL-13 signaling, and the immune system by targeting FCER2, IL4R, FASLG, TGFB1, IL6R, STAT6, IL1B, CASP3, IL5RA, IL2RB, MYD88, SQSTM1, IL12RB1, IFNGR1, ADAM17, and CDH1, thereby regulating pneumonia.

\section{Data Availability}

The data used to support the findings of this study are included within the article.

\section{Conflicts of Interest}

The authors declare that they have no competing interests.

\section{Authors' Contributions}

Yan $\mathrm{Xu}$, Jiali Cai, and Weibin Li conceived and designed the study. Xinna Wang, Hanying Xu, Jingkun Miao, Yan Mei, and Qixiong Chen collected the data. Fang Liu and Hongtao Cui wrote the manuscript. All of the authors were responsible for reviewing the data. All of the authors read and approved the final manuscript. Yan Xu, Jiali Cai, and Weibin Li-contributed equally to this work.

\section{Acknowledgments}

This work was supported by the project funded by China Postdoctoral Science Foundation (2020M673158); the Postdoctoral Special Foundation Grant in 2019 by Chongqing Bureau of Human Resources and Social Security ([2019] No. 298); the Innovation and Application of Traditional Chinese Medicine Technology by Chongqing Science and Technology Bureau and Chongqing Health Commission (No. 2020ZY024084 and 2021MSXM317); the Chongqing Science and Technology Commission Social Livelihood Science and Technology Special Project (Cstc2017shmsA130001); and the Key Projects of the Chongqing Municipal Health Commission (2019ZY013201).

\section{Supplementary Materials}

1074 differentially expressed genes in pneumonia. (Supplementary Materials)

\section{References}

[1] H. Wenjie, C. Tingting, and Z. Hongyan, "Application of bubble blowing combined with lung acupoint massage in the nursing of children with pneumonia," Electronic Journal of Practical Clinical Nursing, vol. 1, no. 12, pp. 181-182, 2016.

[2] J. Hongxia, "Observation of curative effect and nursing analysis of pulmonary acupoint application of Chinese herbal medicine in treating children with pneumonia and coughing," Chinese and Foreign Medical, vol. 38, no. 21, pp. 149-151, 2019.

[3] D. Zhihua, "Clinical study of Yekening paste application in the treatment of mycoplasma pneumonia in children (windheat closed lung)," Chinese Graduate Dissertation, 
Changchun University of Chinese Medicine, Changchun, China, 2014.

[4] L. Zhihong, W. Yanwei, T. Xiaoge, N. Min, and Y. Suping, "Clinical observation on the nursing of 75 cases of infantile pneumonia and cough by mechanical phlegm removal combined with iontophoresis on Feishu," Journal of Pediatrics of Traditional Chinese Medicine, vol. 14, no. 5, pp. 34-36, 2018.

[5] W. Mingjie, Z. Xiuzhuo, and Y. Jun, "Moxibustion assisted treatment of 7 cases of novel coronavirus pneumonia," Chinese Acupuncture, vol. 40, no. 10, pp. 1035-1036, 2020.

[6] Z. Xixin, Z. Zhao, and C. Xin, "Attached to the Essence and Function of the Meridian of the Human Fascial System, Chinese Acupuncture and Moxibustion Society," in Proceedings of the 2017 World Acupuncture and Moxibustion Academic Conference and 2017 Chinese Acupuncture and Moxibustion Society Annual Conference, pp. 715-716, Xi'an, China, 2017.

[7] H. Junyu, "Acupoints, meridians and essential hypothesis of acupuncture," Asia Pacific Traditional Medicine, vol. 8, no. 11, pp. 177-178, 2012.

[8] Q. Hongzhong and L. Ruiqiong, "Phenomenological analysis of the essence of meridians," Chinese Medicine Research, vol. 26, no. 12, pp. 61-64, 2013.

[9] C. M. Michaud, C. J. Murray, and B. R. Bloom, "Burden of disease-implications for future research," JAMA, vol. 285, no. 5, pp. 535-539, 2001.

[10] A. C. Rider and B. W. Frazee, "Community-acquired pneumonia," Emergency Medicine Clinics of North America, vol. 36, no. 4, pp. 665-683, 2018.

[11] I. Rudan, C. Boschi-Pinto, Z. Biloglav, K. Mulholland, and H. Campbell, "Epidemiology and etiology of childhood pneumonia," Bulletin of the World Health Organization, vol. 86, no. 5, pp. 408-416, 2008.

[12] T. Barrett, S. E. Wilhite, P. Ledoux et al., "NCBI GEO: archive for functional genomics data sets-update," Nucleic Acids Research, vol. 41, no. Database issue, pp. D991-D995, 2013.

[13] L. Nana, "Effect of moxibustion "Feishu" and "Xinshu" on cardiac function and myocardial cell autophagy in CHF rats," Chinese Graduate Dissertation, Anhui University of Traditional Chinese Medicine, Hefei, China, 2018.

[14] J. Xuezhao, "The effect of mild moxibustion "Feishu" and "Xinshu" on the expression levels of autophagy-related proteins LC3 and Beclin-1 in CHF rats," Chinese Graduate Dissertation, Anhui University of Traditional Chinese Medicine, Hefei, China, 2017.

[15] L. Haitao, "Study on the effect of acupuncture at "Feishu" point on IL-4, IL-6, IFN- $\gamma$ and ACTH in rats with pulmonary immune inflammation," Chinese Graduate Dissertation, Liaoning University of Traditional Chinese Medicine, Shenyang, China, 2016.

[16] L. Dan, "Effect of moxibustion "Feishu" and "Xinshu" on the expression levels of Bcl-2, Bax, Fas and Fasl related to cardiomyocyte apoptosis in CHF rats," Chinese Graduate Dissertation, Anhui University of Traditional Chinese Medicine, Hefei, China, 2018.

[17] L. Yuli, Z. Lide, L. Haitao et al., "The effect of "Feishu" point on interleukin- $1 \beta$ and interleukin- 6 in lung tissue of bronchial asthma," Progress in Anatomy, vol. 23, no. 6, pp. 565-568, 2017.

[18] X.-F. Zhang, J. Zhu, W.-Y. Geng et al., "Electroacupuncture at Feishu (BL13) and Zusanli (ST36) down-regulates the expression of orexins and their receptors in rats with chronic obstructive pulmonary disease," Journal of Integrative Medicine, vol. 12, no. 5, pp. 417-424, 2014.
[19] C. Li, Z. Yan, L. Jing et al., "Experimental study on moxibustion of "Feishu and Gaolingshu" on E-cad gene expression in lung epithelial cells of rats with pulmonary fibrosis induced by BLMA5," Liaoning Journal of Traditional Chinese Medicine, vol. 44, no. 8, pp. 1762-1765, 2017.

[20] W. Qiang, Z. Yonglei, W. Fengqin et al., "Moxibustion of "Feishu" and "Xinshu" on the expression of myeloid differentiation factor and caspase- 3 in myocardial tissue of rats with chronic heart failure," Acupuncture Research, vol. 41, no. 5, pp. 429-434, 2016.

[21] L. Lisha, C. Zedong, C. Yiguo, L. Chunri, Z. Zhixing, and Z. Xiaoqing, "Effect of acupuncture combined with paclitaxel on the expression of $\mathrm{P}$ glycoprotein, a factor related to apoptosis in mouse lung squamous carcinoma cells," Chinese Journal of Traditional Chinese Medicine, vol. 32, no. 11, pp. 2671-2673, 2014.

[22] L. Lisha, "Effect of acupuncture at Feishu and lingtai points in combination with anticancer drug paclitaxel on the expression of CYP3A and P-GP proteins in mice with lung cancer," Chinese Graduate Dissertation, Liaoning University of Traditional Chinese Medicine, Shenyang, China, 2014.

[23] Y. J. Du Chuanhai and Z. Z. Qiu Liying, "Effect of acupuncture treatment on serum IL-12, IL-4 and IgE levels in patients with asthma," Shandong Journal of Traditional Chinese Medicine, vol. 7, pp. 417-418, 2005.

[24] Z. M. Li Rong, L. F. Li Cuixia, C. Y. Li Wenjun, L. Q. Li Zhigang, and L. Mingfang, "Effect of moxibustion "Feishu" and "Gou Leng" on the balance regulation of type I;/II; cytokines in rats with bleomycin A5 induced pulmonary fibrosis," Acupuncture Research, vol. 3, pp. 164-166, 2005.

[25] G. Yan, "Effect of moxibustion on Feishu on immune substances of different organs in rats with LPS-induced inflammation," Chinese Graduate Dissertation, Beijing University of Chinese Medicine, Beijing, China, 2009.

[26] L. Fuhong, "Experimental study on the effect of moxibustion at Feishu and Gaolingshu on IFN- $\gamma$ in lung tissue of rats with bleomycin A5 induced pulmonary fibrosis," Chinese Graduate Dissertation, Chengdu University of Traditional Chinese Medicine, Chengdu, China, 2006.

[27] L. F. Li Rong, C. Y. Li Wenjun, L. Q. Li Zhigang, Z. M. Li Cuixia, and L. Mingfang, "Experimental study on the effect of moxibustion at Feishu and Gaolingshu on interferon- $\gamma$ in lung tissue of rats with pulmonary fibrosis induced by BLM_ (A5)," New Chinese Medicine, vol. 11, pp. 94-96, 2005.

[28] L. W. Li Rong, L. Z. Cai Yongning, Z. M. Luo Quanan, L. F. Li Cuixia, and L. Mingfang, "Experimental study on moxibustion of "Feishu" and "Gou Leng" on BLM_(A5)-induced TGF- $\beta 1$ expression in rats with pulmonary fibrosis," Chinese Acupuncture, vol. 11, pp. 42-44, 2005.

[29] L. Jing, "Study on the regulating effect of moxibustion on Feishu and Gaolingshu on Wnt/ $\beta$-catenin signal pathway in rats with pulmonary fibrosis," Chinese Graduate Dissertation, Chengdu University of Traditional Chinese Medicine, Chengdu, China, 2017.

[30] H. Xiaojing, S. Suju, H. Jinshuang et al., "Effect of acupuncture "Feishu," "Dazhui" and "Fengmen" on the expression of CC chemokine ligand 1 and CC chemokine receptor 8 in lung tissue of asthmatic rats," Acupuncture Research, vol. 45, no. 5, pp. 363-367, 2020.

[31] Y.-L. Liu, L.-D. Zhang, T.-M. Ma et al., "Feishu acupuncture inhibits acetylcholine synthesis and restores muscarinic acetylcholine receptor M2 expression in the lung when treating allergic asthma," Inflammation, vol. 41, no. 3, pp. 741-750, 2018. 
[32] H. Mengqi, P. Sha, R. Yue, S. Zhan, Z. Yanling, and Q. Yanjiang, "Discovery and application of Chinese medicine efficacy markers based on systemic Chinese medicine," Chinese Journal of Traditional Chinese Medicine, vol. 45, no. 14, pp. 3245-3250, 2020.

[33] G. Puyang, Q. Jin, and Y. Boyang, "Research on the material basis of modern medicinal effects based on the traditional efficacy of Chinese medicine is the source of original research," World Science and Technology-Modernization of Traditional Chinese Medicine, vol. 19, no. 9, pp. 1413-1418, 2017.

[34] J. Rui and Z. Bing, "The cognition of traditional Chinese medicine in the sense of system emergence," Chinese Journal of Traditional Chinese Medicine, vol. 30, no. 4, pp. 977-979, 2015.

[35] G. Jifa and S. Wuqi, "System science and TCM methodology," System Engineering Theory and Practice, vol. 31, no. S1, pp. 24-31, 2011.

[36] Z. Linli, W. Yan, and L. Li, "Research progress of cytokines and inflammatory and immune diseases," Pharmaceutical and Clinical Research, vol. 28, no. 3, pp. 202-205, 2020.

[37] Y. Yang, "The role of interferon gamma and interleukin 4 in chronic obstructive pulmonary disease," Chinese Graduate Dissertation, Xinxiang Medical College, Xinxiang, China, 2018.

[38] X. Yaofang, L. Cuiqing, S. Huiju, and M. Li, "Monitoring of IL-4 and IL-13 levels in airway lavage fluid in children with neonatal respiratory distress syndrome and ventilator-associated pneumonia," Chinese Journal of Contemporary Pediatrics, vol. 11, no. 8, pp. 645-648, 2009.

[39] G. Xuejun, "163 cases of black children bronchial pneumonia were treated by Feishu point application," Shanghai Journal of Traditional Chinese Medicine, vol. 8, p. 39, 2003.

[40] C. Y. Zhang Xin, "Treatment of 28 children with pneumonia by cupping at Feishu point," Journal of Baotou Medical College, vol. 4, p. 332, 2001.

[41] G. Jing, "Clinical observation on the treatment of infantile pneumonia by combining traditional Chinese and western medicine injection at Feishu point," Maternal and Child Health Care in China, vol. 16, pp. 2250-2251, 2006.

[42] W. Luo, J. Y. Wang, C. L. Liu, and C. Huang, "[Effect of electroacupuncture stimulation of "Feishu" (BL 13) on lung index, serum and lung IL-10 and TNF-alpha levels in mice with viral pneumonia]," Zhen Ci Yan Jiu=Acupuncture research, vol. 39, no. 4, pp. 293-297, 2014.

[43] E. M. Katsuya, M. A. Pedreira de Castro, C. R. Whitaker Carneiro, Y. Yamamura, and V. L. F. Silveira, "Acupuncture reduces immune-mediated pulmonary inflammatory lesions induced in rats," Forschende Komplementärmedizin/Research in Complementary Medicine, vol. 16, no. 6, pp. 413-416, 2009.

[44] L. Huiping, "The safety of Chinese herbal hot compress on Feishu acupoints combined with Chinese herbal decoction in the treatment of children with cold and cough," Medical Diet and Health, vol. 18, no. 13, pp. 24-25, 2020.

[45] M. Lan, "Observation on curative effect of Feishu point application of traditional Chinese medicine in treating infantile pneumonia and cough," Inner Mongolia Traditional Chinese Medicine, vol. 39, no. 4, pp. 135-136, 2020.

[46] S. Shuangxiu, "Effect of Chinese medicine iontophoresis combined with massage on Feishu points on curative effect and immune function of children with mycoplasma pneumonia," Chinese Folk Remedies, vol. 27, no. 9, pp. 28-30, 2019.

[47] Q. Hailiang, Joy, C. Zhikun, W. Shiyou, S. Jinmei, and C. Rixin, "Clinical observation on moxibustion at Feishu point for acute attack of children's asthma with cold asthma," Journal of Nanjing University of Traditional Chinese Medicine, vol. 33, no. 4, pp. 363-366, 2017.

[48] H. Yue, S. Jia, W. Lili, Z. Yuan, G. Lirong, and Y. Jianbo, "The role of PI3K/Akt/Nrf2 signaling pathway in the reduction of acute lung injury induced by endotoxic shock in rabbits with electroacupuncture," Chinese Journal of Integrated Traditional Chinese and Western Medicine Surgery, vol. 24, no. 2, pp. 197-202, 2018.

[49] M. Xin, Z. Qiongqiong, X. Xiqing, L. Xuhao, and Y. Jiguo, "Study on the rules of acupoint application for the treatment of allergic rhinitis," World Science and Technology-Modernization of Traditional Chinese Medicine, vol. 21, no. 4, pp. 731-737, 2019.

[50] X. Wei, "Preliminary study on blood perfusion volume and body surface temperature of Feishu points in patients with COPD," Bright Chinese Medicine, vol. 35, no. 13, pp. 19491951, 2020.

[51] Z. Mengxue and G. Ling, "Effect of wheat-grain moxibustion at Zusanli and Feishu points on neutrophil-lymphocyte ratio and platelet count in patients with non-small cell lung cancer after chemotherapy: a randomized controlled study," Chinese Journal of Clinicians, vol. 44, no. 1, pp. 42-45, 2016.

[52] D. Dong and C. Weiqiang, "Observation on therapeutic effect of acupuncture and interventional thermochemotherapy on non-small cell lung cancer," Shaanxi Traditional Chinese Medicine, vol. 35, no. 8, pp. 1063-1065, 2014.

[53] W. Zheng and C. Xiaojun, "Clinical observation of adrenaline on Feishu points through skin administration to assist bronchiolitis," Practical Integrated Chinese and Western Medicine Clinic, vol. 2, pp. 9-10, 2006.

[54] W. Qian, Z. Yonglei, W. Jie et al., "Clinical observation of moxibustion on Feishu and Xinshu in treating chronic heart failure," Shanghai Journal of Acupuncture, vol. 31, no. 2, pp. 91-93, 2012.

[55] C. Fumei and L. Xingqiong, "Observation on therapeutic effect of catgut embedding at acupoints in treating 50 cases of chronic urticaria," Chinese Medicine Guide, vol. 12, no. 26, p. 146, 2014.

[56] L. Q. Ding Renming, “38 cases of herpes zoster treated by moxibustion of Feishu points with ginger," Yunnan Journal of Traditional Chinese Medicine, vol. 4, pp. 24-25, 2003.

[57] F. Yongxi, "Clinical observation on 56 cases of abdominal addiction treated by Feishu and Geshu point acupuncture and cupping," Journal of Clinical Acupuncture, vol. 3, p. 7, 1995.

[58] Z. Jie and L. Donghua, "Clinical observation on treatment of 156 cases of mild and moderate acne with drugs and autoblood therapy," Journal of Nantong University (Medical Edition), vol. 34, no. 3, pp. 242-243, 2014. 